\title{
Comparison of the factors associated with preference of life-sustaining treatment by caregivers' and health facility tenants' families: a cross-sectional study
}

\section{Fumio Shaku ( $\sim$ shaku-gi@umin.ac.jp )}

Nihon University https://orcid.org/0000-0002-5544-0674

Madoka Tsutsumi

Himawari Clinic

Asumi Nakamura

Himawari Clinic

Hiroshi Takagi

Mizonokushi Family Clinic

Takahiro Otsuka

Aketo Otsuka Clinic

Shuicihro Maruoka

Itabashi Hospital, Nihon University

Research article

Keywords: dvance care planning, life-sustaining treatment, home caregiving, old-age health facility

Posted Date: February 28th, 2020

DOI: https://doi.org/10.21203/rs.3.rs-15510/v1

License: (c) (i) This work is licensed under a Creative Commons Attribution 4.0 International License.

Read Full License 
1 Comparison of the factors associated with preference of life-sustaining treatment by

Authors

5 Fumio Shaku ${ }^{\mathrm{a}^{*}}$ M.D., Ph.D, Madoka Tsutusmi ${ }^{\mathrm{b}}$ MPH, Asumi Nakamura ${ }^{\mathrm{b}}$ M.D., Hiroshi

$6 \quad$ Takagi $^{\mathrm{c}}$ M.D., Ph.D., Takahiro Otsuka ${ }^{\mathrm{d}}$ M.D., Shuicihro Maruoka ${ }^{\mathrm{a}}$ M.D., Ph.D.

7 Affiliations

8 a Department of Psychosomatic Internal Medicine, Itabashi Hospital, Nihon University,

9 Tokyo, Japan

10 30-1,Oyaguchikamicho, Itabashi-ku, Tokyo,1738610, Japan

11 bimawari Home Clinic, Chiba, Japan

12 5-1-2, Yurinokidai, Yachiyoshi, Chiba, 1760042, Japan

13 'Mizonokuchi Family Clinic, Kanagawa, Japan

14 3-14-1,Hisamoto, Takatsu-ku, Kawasaki-shi, 2130011, Kanagawa, Japan

15 d Aketo Ohtsuka Clinic, Saitama, Japan

16569, Kawahara-aketo, Kumagaya-shi, 3600837, Saitama

17 *Corresponding author

18 Fumio Shaku

19 Department of psychosomatic internal medicine, Itabashi Hospital, Nihon University

20 30-1, Oyaguchikamicho, Itabashiku, Tokyo, 173-8610, Japan 
Email: shaku-gi@umin.ac.jp

\section{Abstract}

Background: Recently, advance care planning (ACP) in palliative care has been attracting attention in Japan. The Japanese counterpart was established by the Ministry of Health, Labor and Welfare in November 2018. Moreover, discussions on end-of-life treatment are important part of ACP It is suggested that individual feelings at the end-of-life are different; ACP depends on various situations. Decision making by patients themselves is recommended; however, there are cases where patients with dementia or other disorders cannot make decisions by themselves. Health providers may contact surrogates and consider their background for better ACP. We focused on patents' families and examined factors related to the preferences of home caregivers' and old-age health facility tenants' families on patient ACP, including life-sustaining treatment (LST) at end-of-life.

Methods: This cross-sectional study involved home caregivers' and old-age health facility tenants' families in Japan. We distributed 925 self-reported questionnaires comprising items such as number of people living together, comprehension of doctor's explanations, nursing care level and duration, the Patient Health Questionnaire (PHQ)-9 and Short Form (SF)-8, and caregivers' LST preference.

Results: A valid response was obtained from 619 respondents [242 men and 377 women (309 in the home-care group, response rate $=61.1 \% ; 310$ in the facility-care group, response rate $=$ $74.0 \%)]$. The mean age of the respondents was $65.29 \pm 12.09$ years in the home-care group and $63.24 \pm 10.39$ years in the facility-care group. LST preference was significantly 
45 associated with sex, number of people living together, care duration, and comprehension of

46 doctors' explanations in the home-care group but was not significantly associated in the

47 facility-care group. Furthermore, PHQ-9/SF-8 scores were not significantly associated with

48 LST preference of the groups.

49 Conclusions: Factors associated with decision on ACP differed between home caregivers'

50 families and health facility tenants' families. In ACP, healthcare providers should be aware of 51 the nature of each patient's caregiving situation and living environment when discussing LST 52 preference.

53

54 Keywords: advance care planning, life-sustaining treatment, home caregiving, old-age health 55 facility

56

57

58 


\section{Background}

60 Advance care planning (ACP) interventions help elicit a person's wishes for and maintain

61 control over their end-of-life (EOL) care to ensure that those wishes are respected [1]. ACP

62 has been attracting attention in Japan in recent years, and the Japanese counter-part was

63 established by the Ministry of Health, Labor and Welfare in November 2018. Moreover,

64 discussions on EOL treatment are also an important part of ACP. Despite widespread endorsement of ACP, few people discuss future treatment wishes with loved ones and healthcare professionals or record them in an advance directive [2-4]. Previous studies have found a mismatch between patient preferences and the care provided at the EOL when healthcare professionals make the decision about care [5, 6]. A systematic review of ACP highlighted that people with dementia are far less likely to have any form of ACP [7]. Therefore, surrogates are frequently called upon to make healthcare decisions on behalf of the patient.

It has been revealed that there is considerable complexity in undertaking ACP in the context of dementia [8]. When a person has moderate-to-severe dementia, it is difficult to assess whether they have decision-making capacity for advance directives and other EOL care.

For several surrogates, this experience has a negative emotional effect [9]. Surrogates are expected to use their judgment concerning the type of decisions that the patient would prefer on the basis of their knowledge of the patients' values and preferences [9, 10]. In addition, numerous studies have shown substantial inaccuracy in the proxy predictions of patient preferences for healthcare [11]. Surrogate decision-makers are often unprepared to apply substituted judgment or the best-interest standard to decision making on behalf of the patient.

81 The surrogate may pursue nonbeneficial treatment because they are unaware of the patient's preferences or grappling with their own complex emotions [12]. In response to situations like 
this, ACP has been promoted as a mechanism to guide families and care providers in making decisions that an incapacitated patient would have made when he or she was still able to [13]. Under such circumstances, there are cases in which elderly patients are cared for at homes and facilities. For families, taking care of a frail older adult may present challenges for both their next of kin and health services [14].

In Japan, several aged patients are in old-age health facilities, such as nursing homes, and it is expected that the number of elderly people in these facilities will increase. As the number of older adults in assisted living facilities (ALFs) increases, the number of people who die in these facilities is also expected to increase. Limited data exist on the number of older adults with advance directives living in ALFs. The findings from one survey of ALFs reported that 48\%-69\% of deceased ALF residents had an advance directive; however, family members reported much higher estimates $(91 \%-97 \%)$ [15]. There are now some data suggesting that ACP interventions in nursing homes and long-term care settings can reduce unnecessary, and sometimes traumatic, hospitalizations of frail older adults $[16,17]$.

Many families are expected to be surrogates for decision making when the intention of patients cannot be adequately expressed due to brain disorders, including dementia. In addition, it is expected that the number of elderly people staying in healthcare facilities will increase, and decision making in various scenarios can be considered. Previous studies [1821] have reported the relationship between ACP and mental health, such as anxiety and depression. We hypothesized that the differences in environmental conditions, relationship with the physician, quality of life (QOL), and mental health among home caregivers' and oldage health facility tenants' families would affect decision making. Therefore, the purpose of this study was to examine factors influencing the preferences of home caregivers' and oldage health facility tenants' families on a patient's ACP. 
Methods

109 This study was approved by the Human Subjects Institutional Review Board of Nihon

110 University Itabashi Hospital Clinical Research Judging Committee (Approval No. RK-

111 151213-3). The study was conducted in accordance with the principles embodied in the

112 Declaration of Helsinki.

113 The purpose and contents of the questionnaires were explained to potential participants.

114 Informed consent was obtained from all participants. Participants were asked to anonymously

115 complete the questionnaire and return it in a sealed envelope to our research partners. If there

116 were one or more questionnaire items that participants did not wish to answer, they were

117 asked to return the questionnaire partly or completely unanswered.

\section{Participants}

We sent questionnaires via mail to the co-operating staff members of two home-visit clinics and four geriatric health service facilities located in six different prefectures. The cooperating staff members distributed the questionnaires to 506 caregivers' and 419 old-age health facility tenants' families.

\section{Instruments}

Each anonymous questionnaire contained an explanation of its purpose and content. The questionnaire [Additional file 1] included items on family demographics, care duration (month), case assessed code, comprehension level of doctor's explanations regarding the patients' conditions and diseases, whether the family had or had not told the patient what 
130 disease he or she had, degree of nursing care, and life-sustaining treatment (LST) preference.

131 The questionnaire package also included the Short Form (SF)-8 [22-24] and Patient Health

132 Questionnaire (PHQ)-9 [25].

133 The following case assessed codes based on the Japanese long-term care insurance system were 134 employed:

135 Necessary support 1: require social support

136 Necessary support 2: require support for life

137 Nursing care 1: require partial care

138 Nursing care 2: require mild care

139 Nursing care 3: require moderate care

140 Nursing care 4: require severe care

141 Nursing care 5: require the most severe care (almost bedridden condition)

PHQ is a self-administered version of the PRIME-MD diagnostic instrument for common mental disorders [25]. PHQ-9 is the depression module that scores the 9 DSM-IV criteria from "0" (not at all) to " 3 " (nearly every day).

SF-8 ${ }^{\mathrm{TM}}$, an eight-item survey that assesses health-related quality of life (HRQOL), is widely

147 used to evaluate allopathic treatment modalities and has been used in Japanese studies [24].

148 This instrument calculated eight health concepts: physical functioning (PF), role physical 149 (RP), bodily pain (BP), general health (GH), vitality (VT), social functioning, role emotional 150 (RE), and mental health (MH). PF, RP, BP, and GH assess the physical aspects of HRQOL and provide a physical component summary, whereas VT (social functioning), RE, and MH 
assess the mental or psychosocial aspects of HRQOL and provide a mental component summary. The scoring was based on Japanese standards, and the scores ranged from 0 to 100; higher scores indicated better QOL. This scoring system is available in the SF- $8^{\mathrm{TM}}$ manual [24] and was provided by the Institute for Health Outcome and Process Evaluation Research.

\section{Data collection}

We asked 925 participants (home-care group, 506; facility-care group, 419) to anonymously complete the questionnaire and return it in a sealed envelope to the co-operating staff members. Our acceptance criteria were as follows: participants should be $\geq 20$ years old, be an integral part of a caregiving family, be able to answer the questions by themselves, and understand the purpose of this study. If there were one or more questionnaire items that participants did not wish to answer, they were asked to return the questionnaire partly or completely unanswered. Our inclusion criterion was that the questionnaire should be attempted by recipients (incomplete entries were accepted). Unresponsive participants and completely unlisted questionnaires were excluded.

A total of 619 returned questionnaires were judged as valid and included in the analyses.

\section{Data analysis}

All analyses were performed by a statistician at the Japan Institute of Statistical Technology using SPSS (ver. 20; IBM, Tokyo, Japan). We divided the participants into four groups: home caregivers (disease name-notified), home caregivers (disease name-NOTnotified), health facility tenants (disease name-notified), and health facility tenants (disease name-NOT-notified); disease name-notified means that the patient was notified their disease 
name, and disease name-NOT-notified means that the patient was not notified their disease name. Further, chi-squared test and t-test were performed to determine significant differences. The data were summarized and analyzed via binominal logistic regression analysis with the home caregivers' and health facility tenants' families' answers of "not prefer" or "prefer" LST as the objective variable, and the regression coefficient of each item and its test result was obtained. The significance level was set at $P<0.05$. The SF-8 scoring was based on the Manual of the SF-8 Japanese Version [24].

\section{Results}

\section{Demographic characteristics}

A valid response was obtained from 619 respondents (response rate $=66.7 \% ; 242$ men and 377 women [309 in the home-care group, response rate $=61.1 \% ; 310$ in the facility-care group, response rate $=74.0 \%])$. The mean age of the respondents was $65.29 \pm 12.09$ years in the home-care group and $63.24 \pm 10.39$ years in the facility-care group. The age among the home-care group was significantly higher $(P=0.026)$ than that among the facility-care group.

The proportion of individuals living alone was significantly higher in the facility-care group $(17.0 \%)$ than in the home-care group $(5.5 \% ; P<0.0001)$.

The mean care duration was $73.63 \pm 95.32$ months in the home-care group and $58.40 \pm 84.78$ months in the facility-care group. The level of comprehension of the explanation by the physician of the patient's condition was "sufficient" in $63.6 \%$ (home-care group) and $66.9 \%$ (facility-care group), "sure" in $27.7 \%$ (home-care group) and $25.9 \%$ (facility-care group), and "not very well" in 5.3\% (home-care group) and $2.6 \%$ (facility-care group), but the difference was not significant. 
There were no significant differences in the age and sex of the patients between the groups, as answered by the participants who had only one patient (this condition applies to a question of dementia symptom). The level of care among the patients was significantly higher in the facility-care group than in the home-care group $(P<0.0001)$. The rate of cancer as a main disease among the patients was significantly higher in the home-care group (5.9\%) than in the facility-care group $(1.7 \% ; P=0.018)$. The rate of dementia symptom among patients was significantly higher in the facility-care group (73.2\%) than in the home-care group $(62.3 \% ; P=0.007)$.

\section{Disease notification}

The rate of disease notification for patients was not significantly different between the groups (home-care group, 67.2\%; facility-care group, 59.7\%).

\section{Preference for decision making}

Table 1 shows the preferences of participants for decision making. The preference for decision making by the patients themselves was significantly higher than that by a family member in the home-care group $(P<0.0001)$. 
220 Table 1. How would you like to decide on life-sustaining treatment?

221 Facility-care group $(P=0.000)$

\section{Care location}

\section{Preference}

Home Facility Total

Patient

89

38

127

$30.9 \%$

$13.1 \%$

$22.0 \%$

Family

110

171

281

$38.2 \%$

$59.2 \%$

$48.7 \%$

Physician

30

28

58

$10.4 \%$

$9.7 \%$

$10.1 \%$

Not sure

51

43

94

$17.7 \%$

$14.9 \%$

$16.3 \%$

Other

8

9

17

$2.8 \%$

$3.1 \%$

$2.9 \%$

288

289

577 
The mean PHQ-9 score of the participants was significantly higher (more depressive) in the home-care group $($ mean $=13.57 \pm 5.34)$ than in the facility-care group $($ mean $=12.48 \pm$ $4.57 ; P=0.012$ by t-test).

Health-related QOL determined by the SF-8 total score of participants was significantly lower in the home-care group than in the facility-care group $(P<0.0001$ by t-test $)$ The scores for the SF-8 subscales (GH, PF, RP, BP, social functioning, MH, and RE) were significantly lower in the home-care group $(P=0.028, P=0.033, P<0.0001, P=0.048, P<0.0001, P=$ 0.001 , and $P<0.0001$, respectively). Only one subscale, vitality, was not significant $(P=$ $0.051)$.

Factor associated with LST preference in disease-notified group

We used binomial logistic regression analysis to analyze the factors associated with the preference for the patient's LST among participants in disease-notified group. There was no significant difference in preference for PHQ-9 and SF-8 among the participants.

The preference for the patient's LST among participants was significantly associated with sex, number of people living together, and comprehension of the doctors' explanations of the patients' conditions in the home-care group (Table 2). The degree of preference for the patient's LST among women participants was significantly smaller than that among men. The degree of preference for the patient's LST among participants significantly reduced with the increase in the number of people living together. The comprehension of the doctor's 
explanation significantly increased the degree of choosing "preference for LST" among

250 participants who selected "not very well" than among those who selected "sufficiently."

251 However, there were no significant associations with any items in the facility-care group

252 (Table 3).

253 Table 2. Relevance of LST preference in the home-care (disease name-notified) group

\section{$P$ value Odds ratio $(95 \% \mathrm{CI})$}

\begin{tabular}{|c|c|c|}
\hline Sex & $0.018^{*}$ & $0.240(0.074-0.786)$ \\
\hline Age & 0.080 & $1.047(0.994-1.103)$ \\
\hline Marital status: & 0.283 & \\
\hline -Unmarried vs. married & 0.899 & $1.132(0.165-7.779)$ \\
\hline -Unmarried vs. divorced & 0.165 & $5.054(0.515-7.779)$ \\
\hline -Unmarried vs. widowed & 0.622 & $2.230(0.092-54.012)$ \\
\hline Number of people living together & $0.034^{*}$ & $0.444(0.209-0.942)$ \\
\hline Care duration & 0.403 & $0.997(0.991-1.004)$ \\
\hline Comprehension degree of doctor's & 0.180 & \\
\hline explanations: & & \\
\hline -Sufficiently vs. some & 0.559 & $1.455(0.414-5.113)$ \\
\hline -Sufficiently vs. not very well & $0.027^{*}$ & $11.937(1.320-107.925)$ \\
\hline
\end{tabular}

$* P<0.05$

255 Table 3. Relevance of LST preference in the facility-care (disease name-notified) group 


\section{$P$ value Odds ratio $(95 \% \mathrm{CI})$}

\begin{tabular}{lll}
\hline Sex & 0.329 & $0.525(0.143-1.918)$ \\
Age & 0.220 & $1.054(0.969-1.147)$ \\
Marital status: & 0.715 & \\
-Unmarried vs. married & 0.259 & $0.378(0.070-2.047)$ \\
-Unmarried vs. divorced & 0.837 & $0.725(0.034-15.51)$ \\
-Unmarried vs. widowed & 0.999 & \\
Number of people living together & 0.874 & $1.043(0.617-1.764)$ \\
\hline Care duration & 0.677 & $1.003(0.990-1.016)$ \\
\hline Comprehension degree of doctor's & & \\
\hline explanations: & 0.956 & \\
\hline
\end{tabular}

We used binomial logistic regression analysis to analyze the factors related to preference

259 for the patient's LST among participants in disease-NOT-notified group. There was no

260 significant difference in preference for the PHQ-9 and SF-8 among the participants. The

261 preference for the patient's LST among participants was significantly associated with the number of people living together and care duration in the home-care group (Table 4). The degree of preference for the patient's LST among participants significantly reduced with the 
264 increase in the number of people living together. As long as the nursing care duration was

265 longer, "preference for LST" among participants became smaller. However, there were no

266 significant associations with any items in the facility-care group (Table 5).

268 Table 4. Relevance of LST preference in the home-care (disease name-NOT-notified) group

\begin{tabular}{|c|c|c|}
\hline & $P$ value & Odds ratio $(95 \% \mathrm{CI})$ \\
\hline Sex & 0.431 & $0.493(0.085-2.858)$ \\
\hline Age & 0.198 & $0.945(0.867-1.030)$ \\
\hline Marital status: unmarried & 0.820 & \\
\hline -Unmarried vs. married & 0.600 & $2.106(0.130-34.192)$ \\
\hline -Unmarried vs. divorced & 0.351 & $5.920(0.142-247.604)$ \\
\hline -Unmarried vs. widowed & 0.871 & $1.412(0.022-90.761)$ \\
\hline Number of people living together & $0.034^{*}$ & $0.331(0.119-0.922)$ \\
\hline Care duration & $0.046^{*}$ & $0.975(0.950-1.000)$ \\
\hline Comprehension degree of doctor's & 0.658 & \\
\hline explanations: & & \\
\hline -Sufficiently vs. some & 0.654 & $1.463(0.276-7.747)$ \\
\hline -Sufficiently vs. not very well & 0.245 & $7.117(0.260-195.135)$ \\
\hline -Sufficiently vs. never & 0.644 & $2.494(0.052-120.192)$ \\
\hline
\end{tabular}


271 Table 5. Relevance of LST preference in the facility-care (disease name-NOT-notified) group

\begin{tabular}{lcc}
\hline & $P$ value & Odds ratio $(95 \%$ CI $)$ \\
\hline Sex & 0.758 & $0.806(0.204-3.181)$ \\
Age & 0.247 & $0.957(0.889-1.031)$
\end{tabular}

Marital status:

-Unmarried vs. married

-Unmarried vs. divorced

-Unmarried vs. widowed

Number of people living together

Care duration

Comprehension degree of doctor's

explanations:

-Sufficiently vs. some
0.819

$0.417 \quad 3.329(0.182-60.886)$

$0.346 \quad 4.666(0.190-$

114.614)

$0.426 \quad 4.279(0.119$

153.431)

$0.069 \quad 0.478(0.216-1.059)$

$0.975 \quad 1.000(0.991-1.009)$

0.363

$0.074 \quad 3.555(0.884-14.302)$

The tendencies of the odds ratios for PHQ-9/SF-8 scores of participants (home-care group, PHQ-9/SF-8 =.916/1.034; facility-care group, PHQ-9/SF-8 = 1.029/.937), indicating a preference for LST, were not significantly different between the groups. 


\section{Discussion}

We hypothesized that the differences in environmental conditions, relationship with the physician, QOL, and MH among home caregivers' and old-age health facility tenants' families would affect decision making. We classified the patients into home-care and facilitycare groups. A home-care group may show greater respect for the patient's autonomy in activities of routine life. The percentage of patients with dementia was higher in the facilitycare group (possibly because families place more severely affected patients in a facility) than in the home-care group, which indicates that there are difficulties in discussing ACP in advance because of dementia. In addition, respondent's age was higher in the home-care group than in the facility-care group, and aging makes care a greater burden. The home-care group had more number of patients with depression and lower QOL, which may be because of the daily care burden. To reduce the burden on caregivers themselves, it may be useful to encourage greater patient autonomy.

With regard to the preference for the patient's LST among participants, the number of people living together, sex, comprehension level of the doctors' explanations of the patients' conditions, and care duration were factors that were significantly associated with LST preference in the home-care group, but there were no significant associations with any factors in the facility-care group. These findings suggest that the home caregiver's preferences are influenced by giving 24-h care, which may increase the caregiver's distress. Longer care duration may reflect increased burden. In addition, the difficulty of care may be higher for home caregivers than for facility caregivers. PHQ-9/SF-8 scores between the home-care and facility-care groups. In the home-care group, 
higher (more depressive) PHQ-9 scores were associated with lower LST preference, whereas in the facility-care group, higher PHQ-9 scores were associated with higher LST preference. The relationships between the SF-8 score and LST preference were also different between the home-care and facility-care groups. In the home-care group, higher SF-8 scores were associated with higher LST preference, whereas in the facility-care group, higher SF-8 scores were associated with lower LST preference. These results indicate that the factors influencing ACP differ depending on the patient's living environment and extent of care needed. The concept of ACP refers to a wide range of discussions that may include conversations about sustaining therapy [26].

A substantial majority of patients at EOL cannot make treatment decisions for themselves owing to their physical deterioration or mental incapacity [9, 27-31]. In addition, caregivers worldwide prefer LSTs more than terminally ill cancer patients [27, 32-36]. A patient's healthcare experience, including the use of intensive life-sustaining procedures, may affect the health and well-being of family members [37, 38]. Surrogate decision making for seriously/terminally ill patients has been shown to be a challenging and emotionally tumultuous task [9]. Accurately understanding patients' preferences for EOL care can buffer the psychological burden of surrogate decision making [9, 39]; however, surrogates frequently do not accurately know their loved ones' treatment preferences [11]. With regard to the types of surrogates, Ouslander, et al. [40] reported that compared with healthcare providers, family members make more accurate decisions, and Parks, et al. [41] reported that compared with other relations, spouses make more accurate decisions. Furthermore, Schmid, et al. [42] suggested that compared with White surrogates, African-American surrogates may make more accurate decisions. Without accurately understanding patients' EOL care 
preferences, family members and other surrogates often project their own preferences to shape the EOL care actually received by terminally ill patients [43-46]. Family caregivers of individuals with serious illness who undergo intensive life-sustaining medical procedures at EOL may be at risk of negative consequences, including depression [47]. Compared with the spouses of patients not undergoing intensive life-sustaining procedures, surviving spouses of those undergoing such procedures at EOL experience a greater increase in depressive symptoms [10]. Boerner, et al. [48] reported that the families that are best equipped to make collaborative decisions about EOL care and the distress associated with bereavement are precisely individuals who engage in ACP in the first place and have a high level of family functioning. Conversely, people who exhibit low levels of family functioning, including problematic decision making and communication styles, are the least likely to engage in ACP. There are families with patients, such as those with dementia, who cannot make decisions on their own, and ACPs are often required for unavoidable reasons. In a study on a similar theme as ours, Poppe, et al. [49] reported the importance of ACP for people with dementia and their family caregivers and noted that especially for patients in a facility suffering from dementia, discussions with the patient's family about future ACP is required. In addition, we actually investigated the difference in LST preference between the facilitycare patients' and home caregivers' families. members who may not know the patient's preferences or who may disagree with one another or with healthcare providers about an appropriate course of care [48]. In a study on surrogate decision-makers' understanding of dementia patients using semistructured interviews with family members, many participants did not prefer the patients being kept alive by "machines" or "extraordinary measures" for EOL care [50]. Winter and Parks [51] reported that LST preference depends on patients' condition; surrogates of healthy elders preferred more LST 
compared with the surrogates of poor health elders. Moreover, stronger preferences for LST were expressed by non-Whites [51]. This study included 202 surrogates and non-Whites including 63 African-Americans and three individuals who indicated no primary racial group. In our study, 619 participants were analyzed and all participants were Asian (Japanese). In addition, our study included not only home caregivers but also families with patients in facilities and compared the LST preferences of both of them. This revealed differences in the factors associated with LST preferences, depending on where patients were cared for, among those in need.

Considering various social and psychological aspects when healthcare providers talk about ACP, patients and their families may appreciate a provider's recommendations for care that are based on family and patient input, when possible. Healthcare providers need to make efforts to make better ACP possible considering various circumstances of patients and their families.

This study had certain limitations. Our study areas were broadly distributed throughout Japan (northern, eastern, and western), but the attitudes of the participants in these areas (called wards or municipalities) may differ from those of participants in rural areas. Levinson, et al. [52] found that elderly patients poorly understood the concept of resuscitation and predominantly relied on television and print media to define their understanding; further, this understanding may be inconsistent with their actual values and preferences. In addition, many people may not directly face LST situations, and individual understandings of LST may differ. This study was based on a self-reported questionnaire; therefore, further validation of

372 the items, such as by conducting a quantitative study, is required to obtain more detailed 373 information. 

progresses, mental problems, such as dementia and physical troubles, increase, and the patient's understanding ability may be an issue. Owing to the results of our study on ACP among patients' families, factors influencing ACP among the families were clarified among them; furthermore, a considerable difference was observed depending on the environment in which the patient lived. home-care, physical and mental burden is high. Moreover, a significant difference in consciousness toward ACP was observed. Compared with the facility-care group, the homecare group is considered affected by ACP owing to various factors, and we believe that it is necessary for medical providers to recognize this situation. Thus, the findings of this study suggest that healthcare providers need to discuss ACP with the patient's family to provide better support for ACP.

\section{Conclusions}

There are different opinions about ACP between home caregivers' and old-age health facility tenants' families. Healthcare providers need to consider the type and extent of a patient's care and their living environment when discussing ACP with a patient's family.

\section{List of abbreviations}

394 ACP: Advance care planning

395 ALF: Assisted living facilities 
HRQOL: Health-related quality of life

LST: Life-sustaining treatment

401

MH: Mental health

402

PF: Physical functioning

403

QOL: Quality of life

404

RE: Role emotional

405

RP: Role physical

406

407

\section{Declarations}

- Ethics approval and consent to participate: This study was approved by the Human Subjects Institutional Review Board of Nihon University Itabashi Hospital Clinical Research Judging Committee (Approval No. RK-151213-3). The study was conducted

- Consent for publication: Informed consent was obtained from all participants.

- Availability of data and materials: All data generated or analyzed during this study are included in this published article [and its supplementary information files].

- Competing interests: The authors declare that they have no competing interests. 
- Funding: This work was supported by the Yuumi Memorial Foundation for Home Health Care, Japan [grant number 2016-25].

- Authors' contributions: FS was the project leader, made questionnaire and wrote the article.MT, AN, HT, and TO came up with study idea and distributed questionnaires to the participants at their working affiliation.MS provided advice related to contents of the article.

3. Daveson BA, Bausewein C, Murtagh FE, Calanzani N, Higginson IJ, Harding R, et al. 
440 involvement in decision-making involving mental capacity (competency) within $441 \quad$ Europe. Palliat Med. 2013; 27:418-27.

442 4. Halpern SD, Emanuel EJ. Can the United States buy better advance care planning? Ann $443 \quad$ Intern Med. 2015; 162:224-5.

444 5. Rubenfeld GD. Principles and practice of withdrawing life-sustaining treatments. Crit $445 \quad$ Care Clin. 2004; 20:435-51.

446 6. Shanawani H, Wenrich MD, Tonelli MR, Curtis JR. Meeting physicians' 447 responsibilities in providing end-of-life care. Chest. 2008; 133:775-86.

448 7. Lovell A, Yates P. Advance Care Planning in palliative care: A systematic literature 449 review of the contextual factors influencing its uptake 2008-2012. Palliat Med. 2014; $450 \quad 28: 1026-35$.

8. Ryan T, Amen KM, McKeown J. The advance care planning experiences of people with dementia, family caregivers and professionals: A synthesis of the qualitative literature. Ann Palliat Med. 2017; 6:380-9.

9. Wendler D, Rid A. Systematic review: the effect on surrogates of making treatment decisions for others. Ann Intern Med. 2011; 154:336-46.

10. Abbott KH, Sago JG, Breen CM, Abernethy AP, Tulsky JA. Families looking back: one year after discussion of withdrawal or withholding of life-sustaining support. Crit Care Med. 2001; 29:197-201. makers: A systematic review. Arch Intern Med. 2006; 166:493-7. 
12. Berlin A, Kunac A, Mosenthal AC. Perioperative goal-setting consultations by surgical colleagues: A new model for supporting patients, families, and surgeons in shared decision making. Ann Palliat Med. 2017; 6:178-82.

13. Bravo G, Trottier L, Arcand M, Boire-Lavigne AM, Blanchette D, Dubois MF, et al. Promoting advance care planning among community-based older adults: A randomized controlled trial. Patient Educ Couns. 2016; 99:1785-95.

14. Shiba K, Kondo N, Kondo K. Informal and formal social support and caregiver burden: the AGES caregiver survey. J Epidemiol. 2016; 26:622-8.

15. Daaleman TP, Williams CS, Preisser JS, Sloane PD, Biola H, Zimmerman S. Advance care planning in nursing homes and assisted living communities. J Am Med Dir Assoc. 2009; 10:243-51.

16. Brinkman-Stoppelenburg A, Rietjens JA, van der Heide A. The effects of advance care planning on end-of-life care: A systematic review. Palliat Med. 2014; 28:1000-25.

17. Graverholt B, Forsetlund L, Jamtvedt G. Reducing hospital admissions from nursing homes: A systematic review. BMC Health Serv Res. 2014; 14:36.

18. Chiarchiaro J, Buddadhumaruk P, Arnold RM, White DB. Prior advance care planning is associated with less decisional conflict among surrogates for critically ill patients. Ann Am Thorac Soc. 2015; 12:1528-33.

19. Song MK, Ward SE, Fine JP, Hanson LC, Lin FC, Hladik GA, et al. Advance care planning and end-of-life decision making in dialysis: A randomized controlled trial targeting patients and their surrogates. Am J Kidney Dis. 2015; 66:813-22. 
20. Schulz R, Boerner K, Klinger J, Rosen J. Preparedness for death and adjustment to bereavement among caregivers of recently placed nursing home residents. J Palliat Med. 2015; 18:127-33.

21. Garrido MM, Prigerson HG. The end-of-life experience: modifiable predictors of caregivers' bereavement adjustment. Cancer. 2014; 120:918-25.

22. Fukuhara S, Bito S, Green J, Hsiao A, Kurokawa K. Translation, adaptation, and validation of the SF-36 Health Survey for use in Japan. J Clin Epidemiol. 1998; 51:1037-44.

23. Fukuhara S, Ware JE, Kosinski M, Wada S, Gandek B. Psychometric and clinical tests of validity of the Japanese SF-36 Health Survey. J Clin Epidemiol. 1998; 51:1045-53.

24. Fukuhara S, Suzukamo Y. Manual of the SF-8 Japanese version. Kyoto: Institute for Health Outcomes \& Process Evaluation Research; 2004.

25. Kroenke K, Spitzer RL, Williams JB. The PHQ-9: validity of a brief depression severity measure. J Gen Intern Med. 2001; 16:606-13.

26. Dingfield LE, Kayser JB. Integrating advance care planning into practice. Chest. 2017; 151:1387-93.

27. Heyland DK, Barwich D, Pichora D, Dodek P, Lamontagne F, You JJ, et al. (Advance care planning evaluation in elderly patients) study team; Canadian researchers at the end of Life Network (CARENET). Failure to engage hospitalized elderly patients and their families in advance care planning. JAMA Intern Med. 2013; 173:778-87. 
28. Torke AM, Sachs GA, Helft PR, Montz K, Hui SL, Slaven JE, et al. Scope and outcomes of surrogate decision making among hospitalized older adults. JAMA Intern Med. 2014; 174:370-7.

29. Zaros MC, Curtis JR, Silveira MJ, Elmore JG. Opportunity lost: end-of-life discussions in cancer patients who die in the hospital. J Hosp Med. 2013; 8:334-40.

30. Sviri S, Garb Y, Stav I, Rubinow A, Linton DM, Caine YG, et al. Contradictions in end-of-life decisions for self and other, expressed by relatives of chronically ventilated patients. J Crit Care. 2009; 24:293-301.

31. Lee JK, Keam B, An AR, Kim TM, Lee SH, Kim DW, et al. Surrogate decisionmaking in Korean patients with advanced cancer: A longitudinal study. Support Care Cancer. 2013; 21:183-90.

32. Ackroyd R, Russon L, Newell R. Views of oncology patients, their relatives and oncologists on cardiopulmonary resuscitation (CPR): questionnaire-based study. Palliat Med. 2007; 21:139-44.

33. Sonnenblick M, Friedlander Y, Steinberg A. Dissociation between the wishes of terminally ill parents and decisions by their offspring. J Am Geriatr Soc. 1993; 41:599604.

34. Lee J, Kim KH. Perspectives of Korean patients, families, physicians and nurses on advance directives. Asian Nurs Res. 2010; 4:185-93. 
35. Hwang IC, Keam B, Kim YA, Yun YH. Factors related to the differential preference for cardiopulmonary resuscitation between patients with terminal cancer and that of their respective family caregivers. Am J Hosp Palliat Care. 2016; 33:20-6.

36. Malhotra C, Farooqui MA, Kanesvaran R, Bilger M, Finkelstein E. Comparison of preferences for end-of-life care among patients with advanced cancer and their caregivers: A discrete choice experiment. Palliat Med. 2015; 29:842-50.

37. Ornstein KA, Boerner K, Siu AL, Schulz R. Downstream effects of end-of-life care for older adults with serious illness on health care utilization of family caregivers. J Palliat Med. 2015; 18:736-7.

38. Davydow DS, Hough CL, Langa KM, Iwashyna TJ. Depressive symptoms in spouses of older patients with severe sepsis. Crit Care Med. 2012; 40:2335-41.

39. Majesko A, Hong SY, Weissfeld L, White DB. Identifying family members who may struggle in the role of surrogate decision maker. Crit Care Med. 2012; 40:2281-6.

40. Ouslander JG, Tymchuk AJ, Rahbar B. Health care decisions among elderly long-term care residents and their potential proxies. Arch Intern Med. 1989; 149:1367-72.

41. Parks SM, Winter L, Santana AJ, Parker B, Diamond JJ, Rose M, et al. Family factors in end-of-life decision-making: family conflict and proxy relationship. J Palliat Med. $2011 ; 14: 179-84$.

42. Schmid B, Allen RS, Haley PP, Decoster J. Family matters: dyadic agreement in endof-life medical decision making. Gerontologist. 2010; 50:226-37. 
43. Fritsch J, Petronio S, Helft PR, Torke AM. Making decisions for hospitalized older adults: ethical factors considered by family surrogates. J Clin Eth. 2013; 24:125-34.

44. Ishikawa Y, Fukui S, Saito T, Fujita J, Watanabe M, Yoshiuchi K. Family preference for place of death mediates the relationship between patient preference and actual place of death: A nationwide retrospective cross-sectional study. PLOS ONE. 2013;8: e56848.

45. Marks MA, Arkes HR. Patient and surrogate disagreement in end-of-life decisions: can surrogates accurately predict patients' preferences? Med Decis Mak. 2008; 28:524-31.

46. Moorman SM, Carr D. Spouses' effectiveness as end-of-life health care surrogates: accuracy, uncertainty, and errors of overtreatment or undertreatment. Gerontologist. $2008 ; 48: 811-9$.

47. Ornstein KA, Aldridge MD, Garrido MM, Gorges R, Bollens-Lund E, Siu AL, et al. The use of life-sustaining procedures in the last month of life is associated with more depressive symptoms in surviving spouses. J Pain Symptom Manag. 2017; 53:178187.e1.

48. Boerner K, Carr D, Moorman S. Family relationships and advance care planning: do supportive and critical relations encourage or hinder planning? J Gerontol B Psychol Sci Soc Sci. 2013; 68:246-56.

49. Poppe M, Burleigh S, Banerjee S. Qualitative evaluation of advanced care planning in early dementia (ACP-ED). PLOS ONE. 2013;8: e60412. 
561

562

563

564

565

566

567

568

569

50. Black BS, Fogarty LA, Phillips H, Finucane T, Loreck DJ, Baker A, et al. Surrogate decision makers' understanding of dementia patients' prior wishes for end-of-life care. J Aging Health. 2009; 21:627-50.

51. Winter L, Parks SM. Elders' preferences for life-prolonging treatment and their proxies' substituted judgment: influence of the elders' current health. J Aging Health. 2012; 24:1157-78.

52. Levinson M, Ho S, Mills A, Kelly B, Gellie A, Rouse A. Language and understanding of cardiopulmonary resuscitation amongst an aged inpatient population. Supplemental material 1 Psychol Health Med. 2017; 22:227-36. 